\title{
Synthesis of Tungsten Complexes Containing an Intramolecularly Coordinated Alkyne Group
}

Murray V. Baker* and Simon K. Brayshaw

Chemistry M313, School of Biomedical and Chemical Sciences, The University of Western Australia, 35 Stirling Highway, Crawley, WA 6009, Australia

Email: mvb@chem.uwa.edu.au

\section{Supporting Information}

5-Iodo-1-pentyne ${ }^{1}$ and 6-iodo-1-hexyne ${ }^{2}$ were prepared using literature methods. Cyclopentadiene was obtained by the thermal cracking of dicyclopentadiene and redistilled immediately prior to use.

\section{Pent-4-ynylcyclopentadiene.}

This compound was prepared using the general procedure Alder and Ache used for making other alkyl substituted cyclopentadines. ${ }^{3}$ Cyclopentadiene $(5.0 \mathrm{~g}, 76 \mathrm{mmol})$ was added to a solution of sodium $(0.8 \mathrm{~g}, 35 \mathrm{mmol})$ in liquid ammonia $(100 \mathrm{~mL})$ and the mixture was stirred until the blue colour discharged ( $c a .1$ minute). 5-Iodo-1-pentyne $(5.0 \mathrm{~g}, 26 \mathrm{mmol})$ was added and the ammonia was allowed to evaporate slowly overnight. Water $(50 \mathrm{~mL})$ and $\mathrm{NH}_{4} \mathrm{Cl}(5 \mathrm{~g})$ were added and the mixture was extracted with ether $(3 \times 30 \mathrm{~mL})$. The combined ether extracts were washed with $\mathrm{HCl}(0.5 \mathrm{M}, 20$ $\mathrm{mL}), \mathrm{Na}_{2} \mathrm{~S}_{2} \mathrm{O}_{3}(10 \%, 10 \mathrm{~mL}), \mathrm{NaHCO}_{3}(10 \%, 10 \mathrm{~mL})$ and dried $\left(\mathrm{MgSO}_{4}\right)$. The solvent was removed in vacuo and the residue was distilled to give pent-4-ynylcyclopentadiene (mixture of 1- and 2- isomers) as a pale yellow liquid. Yield: $2.65 \mathrm{~g}(78 \%) . \mathrm{bp}_{0.7} 40-45^{\circ}$.

${ }^{1} \mathrm{H}$ NMR (300.13 MHz, $\left.\mathrm{CDCl}_{3}\right): \delta$ 6.0-6.5 (m, 3H, vinylic $\left.\mathrm{Cp} \mathrm{H}\right), 2.88,2.85$ (m, 2H, H5), 2.4-2.6 (m, 
2H, H1'), $2.21\left(\mathrm{td}, \mathrm{J}_{3^{\prime}, 2^{\prime}}=7.1 \mathrm{~Hz}, \mathrm{~J}_{3^{\prime}, 5^{\prime}}=2.7 \mathrm{~Hz}, 2 \mathrm{H}, \mathrm{H} 3^{\prime}\right), 1.97\left(\mathrm{t}, \mathrm{J}_{5^{\prime}, 3^{\prime}}=2.7 \mathrm{~Hz}, 1 \mathrm{H}, \mathrm{H} 5^{\prime}\right), 1.7-1.9(\mathrm{~m}, 2 \mathrm{H}$, $\left.\mathrm{H} 2^{\prime}\right)$.

${ }^{13} \mathrm{C}$ NMR $\left(75.47 \mathrm{MHz}, \mathrm{CDCl}_{3}\right): \delta 148.69,146.14(\mathrm{C}), 134.64,134.02,132.54,130.85,127.03,126.63$ (CH), 84.50, $84.41\left(\mathrm{C}^{\prime}\right)$, 68.61, 68.57 (C5'), 43.35, 41.41 (C5), 29.72, 28.84, 28.52, 27.72 (C1', C2'), $18.22,18.12\left(\mathrm{C}^{\prime}\right)$.

Anal. Calc for $\mathrm{C}_{10} \mathrm{H}_{12}$ : C, 90.85; H, 9.15. Found: C, 91.10; H, 8.88.

\section{Hex-5-ynylcyclopentadiene.}

This compound was prepared as for the pentynyl analogue and was obtained as a colourless liquid (mixture of 1- and 2- isomers). Yield: $75 \% . \mathrm{bp}_{0.1} 40^{\circ}$.

${ }^{1} \mathrm{H}$ NMR (300.13 MHz, $\mathrm{CDCl}_{3}$ ): $\delta$ 5.9-6.5 (m, 3H, vinylic Cp H), 2.93, 2.86 (m, 2H, H5), 2.3-2.5 (m, 2H, H1'), $2.22\left(\mathrm{td}, \mathrm{J}_{4^{\prime}, 3^{\prime}}=6.9 \mathrm{~Hz}, \mathrm{~J}_{4^{\prime}, 6^{\prime}}=2.7 \mathrm{~Hz}, 2 \mathrm{H}, \mathrm{H} 4^{\prime}\right), 1.95\left(\mathrm{t}, \mathrm{J}_{6^{\prime}, 4^{\prime}}=2.7 \mathrm{~Hz}, 1 \mathrm{H}, \mathrm{H} 6^{\prime}\right), 1.5-1.8(\mathrm{~m}, 4 \mathrm{H}$, $\mathrm{H} 2^{\prime}$ and $\left.\mathrm{H} 3^{\prime}\right)$.

${ }^{13} \mathrm{C}$ NMR $\left(75.47 \mathrm{MHz}, \mathrm{CDCl}_{3}\right): \delta 149.57,146.86(\mathrm{C}), 134.76,133.89,132.54,130.69,126.62,126.17$ (CH), 84.68, 84.62 (C5'), 68.41, 68.36 (C6'), 43.31, 41.36 (C5), 30.27, 29.39, 28.81, 28.30, 28.28, $27.97\left(\mathrm{C} 1{ }^{\prime}, \mathrm{C} 2 ', \mathrm{C} 3 '\right), 18.41\left(\mathrm{C} 4{ }^{\prime}\right)$.

Anal. Calc for $\mathrm{C}_{11} \mathrm{H}_{14}$ : C, 90.35; H, 9.65. Found: C, 90.29; H, 9.54.

\section{$\left(\eta^{5}-\mathrm{C}_{5} \mathrm{H}_{4} \mathrm{CH}_{2} \mathrm{CH}_{2} \mathrm{CH}_{2} \mathrm{C} \equiv \mathrm{CH}\right) \mathrm{W}(\mathrm{CO})_{3} \mathrm{Cl}(1)$.}

n-Butyllithium $(0.4 \mathrm{~mL}, 2.0 \mathrm{M}, 0.8 \mathrm{mmol})$ was added to a solution of pent-4-ynylcyclopentadiene $(0.15 \mathrm{~g}, 1.1 \mathrm{mmol})$ in THF $(10 \mathrm{~mL})$ at $-80^{\circ}$, and the mixture was allowed to warm to room temperature over $2 \mathrm{~h}$.

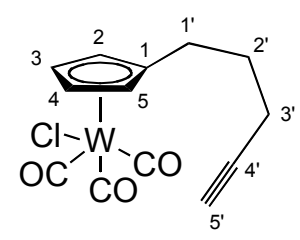

1 Tris(propionitrile)tungsten tricarbonyl $(0.28 \mathrm{~g}, 0.65 \mathrm{mmol})$ was added and the mixture was stirred overnight. Acetic acid $(0.3 \mathrm{~mL})$ and $\mathrm{CCl}_{4}(0.5 \mathrm{~mL})$ were added and the solution was stirred for $1 \mathrm{~h}$, then poured into water $(30 \mathrm{~mL})$ and the mixture was extracted with 
dichloromethane $(2 \times 20 \mathrm{~mL})$. The combined organic extracts were washed with water $(2 \times 10 \mathrm{~mL})$ and dried $\left(\mathrm{MgSO}_{4}\right)$. The solvent was removed in vacuo and the residue was subjected to flash chromatography on silica (30\% ethyl acetate / hexanes) and recrystallised from benzene to give $\mathbf{1}$ as an orange/red solid (0.19 g, 68\%).

HR-MS (FAB): $m / z 435.9952\left(\mathrm{C}_{13} \mathrm{H}_{11}{ }^{35} \mathrm{ClO}_{3}{ }^{186} \mathrm{~W}\right.$ requires 435.9940$)$.

LR-MS (FAB): $m / z$ 348, 349, 350, 352 (matches M-3CO, $\mathrm{C}_{10} \mathrm{H}_{11}{ }^{35} \mathrm{Cl}^{182,183,184,186} \mathrm{~W}$ ).

IR (THF): $v(C O) 2041,1952 \mathrm{~cm}^{-1}$.

Anal. Calc for $\mathrm{C}_{13} \mathrm{H}_{11} \mathrm{ClO}_{3} \mathrm{~W} \cdot 0.2 \mathrm{C}_{6} \mathrm{H}_{6}$ : C, 37.89; H, 2.73. Found: C, 38.01; H, 3.07.

${ }^{1} \mathrm{H}$ NMR (500.13 MHz, $\mathrm{C}_{6} \mathrm{D}_{6}$ ): $\delta 4.72$ (apparent t, splitting $2.2 \mathrm{~Hz}, 2 \mathrm{H}, \mathrm{H} 2 / \mathrm{H} 5$ ), 4.15 (apparent t, splitting 2.2 Hz, 2H, H3/H4), 1.98 (apparent t, splitting $\left.7.8 \mathrm{~Hz}, 2 \mathrm{H}, \mathrm{H} 1^{\prime}\right), 1.8\left(\mathrm{td}, \mathrm{J}_{3^{\prime}, 2^{\prime}}=7.0 \mathrm{~Hz}, \mathrm{~J}_{3^{\prime}, 5^{\prime}}=2.6\right.$ $\left.\mathrm{Hz}, 2 \mathrm{H}, \mathrm{H} 3^{\prime}\right), 1.75$ (t, $\left.\mathrm{J}_{5^{\prime}, 3^{\prime}}=2.6 \mathrm{~Hz}, 1 \mathrm{H}, \mathrm{H} 5^{\prime}\right), 1.3-1.4\left(\mathrm{~m}, 2 \mathrm{H}, \mathrm{H} 2^{\prime}\right)$.

${ }^{13} \mathrm{C}$ NMR (125.8 MHz, $\left.\mathrm{C}_{6} \mathrm{D}_{6}\right): \quad \delta 232.73(\mathrm{CO}), 216.85(2 \times \mathrm{CO}), 125.91(\mathrm{C} 1), 92.07(\mathrm{C} 2 / \mathrm{C} 5), 86.86$ (C3/C4), $83.13\left(\mathrm{C}^{\prime}\right), 69.90\left(\mathrm{C}^{\prime}\right), 28.36,27.60\left(\mathrm{C}^{\prime}{ }^{\prime} / \mathrm{C}^{\prime}\right), 18.10\left(\mathrm{C} 3^{\prime}\right)$.

\section{$\left(\eta^{5}-\mathrm{C}_{5} \mathrm{H}_{4} \mathrm{CH}_{2} \mathrm{CH}_{2} \mathrm{CH}_{2} \mathrm{CH}_{2} \mathrm{C} \equiv \mathrm{CH}\right) \mathrm{W}(\mathrm{CO})_{3} \mathrm{Cl}(2)$.}

A solution of hex-5-ynylcyclopentadiene $(0.11 \mathrm{~g}, 0.75 \mathrm{mmol})$ and potassium tert-butoxide $(0.077 \mathrm{~g}, 0.69 \mathrm{mmol})$ in DMSO $(3 \mathrm{~mL})$ was stirred for $20 \mathrm{~min}$. Tris(propionitrile)tungsten tricarbonyl $(0.24 \mathrm{~g}, 0.55 \mathrm{mmol})$ was added and the

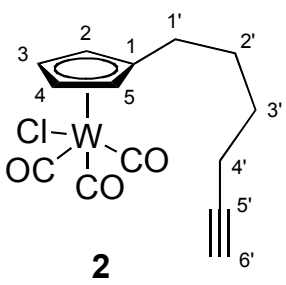
mixture was stirred overnight. Water $(10 \mathrm{~mL})$ was added and the mixture was washed with ether $(2 \times 5$ $\mathrm{mL})$ and hexanes $(2 \times 10 \mathrm{~mL})$, then $\mathrm{HCl}(2 \mathrm{M}, 5 \mathrm{~mL}, 10 \mathrm{mmol})$ and $\mathrm{CCl}_{4}(3 \mathrm{~mL})$ were added and the mixture was stirred for $2 \mathrm{~h}$. The organic layer was separated, washed with water $(5 \mathrm{~mL})$, dried $\left(\mathrm{MgSO}_{4}\right)$, and stripped of solvent under reduced pressure. Chromatography on silica (20\% EtOAc / hexanes) gave 2 as an orange red solid $(0.13 \mathrm{~g}, 53 \%)$.

LR-MS (FAB): $m / z$ 362, 363, 364, 366 (matches M-3CO, $\mathrm{C}_{14} \mathrm{H}_{13}{ }^{35} \mathrm{Cl}^{182,183,184,186} \mathrm{~W}$ )

IR (THF): v(CO) 2040, $1951 \mathrm{~cm}^{-1}$. 
Anal. Calc for $\mathrm{C}_{14} \mathrm{H}_{13} \mathrm{ClO}_{3} \mathrm{~W}:$ C, 37.49; H, 2.92. Found: C, 37.10; H, 3.04.

${ }^{1} \mathrm{H}$ NMR (500.13 MHz, $\mathrm{C}_{6} \mathrm{D}_{6}$ ): $\delta 4.70$ (apparent t, splitting $2.2 \mathrm{~Hz}, 2 \mathrm{H}, \mathrm{H} 2 / \mathrm{H} 5$ ), 4.14 (apparent t, splitting 2.2 Hz, 2H, H3/H4), $1.84\left(\mathrm{td}, \mathrm{J}_{4^{\prime}, 3^{\prime}}=6.6 \mathrm{~Hz}, \mathrm{~J}_{4^{\prime}, 6^{\prime}}=2.7 \mathrm{~Hz}, 2 \mathrm{H}, \mathrm{H} 4^{\prime}\right), 1.80$ (apparent t, splitting 7.5 $\left.\mathrm{Hz}, 2 \mathrm{H}, \mathrm{H} 1^{\prime}\right), 1.77$ (t, $\left.\mathrm{J}_{6}^{\prime}, 4^{\prime}=2.7 \mathrm{~Hz}, 1 \mathrm{H}, \mathrm{H6} 6^{\prime}\right), 1.10-1.23$ (m, 4H, H2' and H3').

${ }^{13} \mathrm{C}$ NMR (125.8 MHz, $\left.\mathrm{C}_{6} \mathrm{D}_{6}\right): \quad \delta 232.89(\mathrm{CO}), 216.88(2 \times \mathrm{CO}), 126.76(\mathrm{C} 1), 91.94(\mathrm{C} 2 / \mathrm{C} 5), 86.72$ (C3/C4), 83.82 (C5'), 69.27 (C6'), 28.77, 28.19, 28.15 (C1', C2', C3'), 18.33 (C4').

\section{$\left(\eta^{5}: \eta^{2}-\mathrm{C}_{5} \mathrm{H}_{4} \mathrm{CH}_{2} \mathrm{CH}_{2} \mathrm{CH}_{2} \mathrm{C} \equiv \mathrm{CH}\right) \mathrm{W}(\mathrm{CO}) \mathrm{Cl}(6)$.}

A solution of $\mathbf{1}$ in $\mathrm{d}_{6}$-benzene in a Pyrex NMR tube cooled in a water bath $\left(c a .25^{\circ} \mathrm{C}\right)$ was irradiated using a $150 \mathrm{~W}$ tungsten lamp. Reaction progress was monitored by ${ }^{1} \mathrm{H}$ NMR spectroscopy and consumption of 1 was complete after 60 min. Yield of $6: 10 \%$ by ${ }^{1} \mathrm{H}$ NMR.

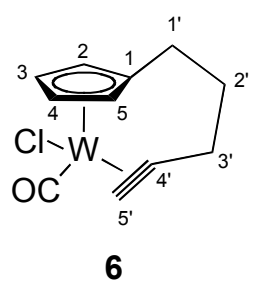

${ }^{1} \mathrm{H}$ NMR (500.13 MHz, $\left.\mathrm{C}_{6} \mathrm{D}_{6}\right): \delta 11.92$ (triplet with ${ }^{183} \mathrm{~W}$ satellites, $\mathrm{J}_{\mathrm{W}-\mathrm{H}}=5.1 \mathrm{~Hz}, \mathrm{~J}_{5^{\prime}, 3^{\prime}}=1.1 \mathrm{~Hz}$ ), 5.71 $\left(\mathrm{dtt}, \mathrm{J}_{2,3}=3.0 \mathrm{~Hz}, \mathrm{~J}_{2,4 / 5}=1.9 \mathrm{~Hz}, \mathrm{~J}_{2,1^{\prime}}=0.5 \mathrm{~Hz}, 1 \mathrm{H}, \mathrm{H} 2\right), 5.25\left(\mathrm{ddd}, \mathrm{J}_{4,3}=3.6 \mathrm{~Hz}, \mathrm{~J}_{4,5}=2.7 \mathrm{~Hz}, \mathrm{~J}_{4,2}=1.8\right.$ $\mathrm{Hz}, 1 \mathrm{H}, \mathrm{H} 4), 5.21\left(\mathrm{dddd}, \mathrm{J}_{3,4}=3.6 \mathrm{~Hz}, \mathrm{~J}_{3,2}=3.0 \mathrm{~Hz}, \mathrm{~J}_{3,4}=2.1 \mathrm{~Hz}, \mathrm{~J}_{3,1^{\prime} \mathrm{a}}=0.6 \mathrm{~Hz}, 1 \mathrm{H}, \mathrm{H} 3\right.$ ), 3.00 (apparent q, splitting 2.2 Hz, 1H, H5), 2.62 (dddd, $\mathrm{J}_{3^{\prime} \mathrm{a}, 3^{\prime} \mathrm{b}}=16.8 \mathrm{~Hz}, \mathrm{~J}_{3^{\prime} \mathrm{a}, 2^{\prime} \mathrm{a}}=11.0 \mathrm{~Hz}, \mathrm{~J}_{3^{\prime} \mathrm{a}, 2^{\prime} \mathrm{b}}=4.8 \mathrm{~Hz}$, $\left.\mathrm{J}_{3^{\prime} \mathrm{a}, 5^{\prime}}=1.0 \mathrm{~Hz}, 1 \mathrm{H}, \mathrm{H} 3^{\prime} \mathrm{a}\right), 2.55$ (ddddd, $\mathrm{J}_{3^{\prime} \mathrm{b}, 3^{\prime} \mathrm{a}}=16.8 \mathrm{~Hz}, \mathrm{~J}_{3^{\prime} \mathrm{b}, 2^{\prime} \mathrm{a}}=5.9 \mathrm{~Hz}, \mathrm{~J}_{3^{\prime} \mathrm{b}, 2^{\prime} \mathrm{b}}=3.0 \mathrm{~Hz}, \mathrm{~J}_{3^{\prime} \mathrm{b}, 5^{\prime}}=1.2 \mathrm{~Hz}, 0.6$ $\left.\mathrm{Hz}, 1 \mathrm{H}, \mathrm{H} 3{ }^{\prime} \mathrm{b}\right), 2.22$ (dddq, $\left.\mathrm{J}_{1^{\prime} \mathrm{a}, 1^{\prime} \mathrm{b}}=14.7 \mathrm{~Hz}, \mathrm{~J}_{1^{\prime} \mathrm{a}, 2^{\prime} \mathrm{b}}=5.6 \mathrm{~Hz}, \mathrm{~J}_{1^{\prime} \mathrm{a}, 2^{\prime} \mathrm{a}}=2.5 \mathrm{~Hz}, \mathrm{~J}_{1^{\prime} \mathrm{a}, 2 / 3 / 5}=0.5 \mathrm{~Hz}, 1 \mathrm{H}, \mathrm{H} 1^{\prime} \mathrm{a}\right), 1.71$ (ddddd, $\left.\mathrm{J}_{2^{\prime} \mathrm{a}, 2^{\prime} \mathrm{b}}=13.8 \mathrm{~Hz}, \mathrm{~J}_{2^{\prime} \mathrm{a}, 1^{\prime} \mathrm{b}}=12.7 \mathrm{~Hz}, \mathrm{~J}_{2^{\prime} \mathrm{a}, 3^{\prime} \mathrm{a}}=11.0 \mathrm{~Hz}, \mathrm{~J}_{2^{\prime} \mathrm{a}, 3^{\prime} \mathrm{b}}=5.9 \mathrm{~Hz}, \mathrm{~J}_{2^{\prime} \mathrm{a}, 1^{\prime} \mathrm{a}}=2.5 \mathrm{~Hz}, 1 \mathrm{H}, \mathrm{H} 2^{\prime} \mathrm{a}\right), 1.48$ (ddddd, $\mathrm{J}_{2^{\prime} \mathrm{b}, 2^{\prime} \mathrm{a}}=13.8 \mathrm{~Hz}, \mathrm{~J}_{2^{\prime} \mathrm{b}, 1^{\prime} \mathrm{a}}=5.6 \mathrm{~Hz}, \mathrm{~J}_{2^{\prime} \mathrm{b}, 3^{\prime} \mathrm{a}}=4.8 \mathrm{~Hz}, \mathrm{~J}_{2^{\prime} \mathrm{b}, 3^{\prime} \mathrm{b}}=3.0 \mathrm{~Hz}, \mathrm{~J}_{2^{\prime} \mathrm{b}, 1^{\prime} \mathrm{b}}=2.4 \mathrm{~Hz}, 1 \mathrm{H}, \mathrm{H} 2^{\prime} \mathrm{b}$ ), 0.28 (ddd, $\left.\mathrm{J}_{1^{\prime} \mathrm{b}, 1^{\prime} \mathrm{a}}=14.7 \mathrm{~Hz}, \mathrm{~J}_{1^{\prime} \mathrm{b}, 2^{\prime} \mathrm{a}}=12.7 \mathrm{~Hz}, \mathrm{~J}_{1^{\prime} \mathrm{b}, 2^{\prime} \mathrm{b}}=2.4 \mathrm{~Hz}, 1 \mathrm{H}, \mathrm{H} 1^{\prime} \mathrm{b}\right)$.

\section{$\left(\eta^{5}: \eta^{2}-\mathrm{C}_{5} \mathrm{H}_{4} \mathrm{CH}_{2} \mathrm{CH}_{2} \mathrm{CH}_{2} \mathrm{CH}_{2} \mathrm{C} \equiv \mathrm{CH}\right) \mathrm{W}(\mathrm{CO}) \mathrm{Cl}(4)$.}

A solution of $\mathbf{2}$ in $\mathrm{d}_{6}$-benzene in a Pyrex NMR tube cooled in a water bath ( $c a$. $25^{\circ} \mathrm{C}$ ) was irradiated using a $150 \mathrm{~W}$ tungsten lamp. Reaction progress was monitored

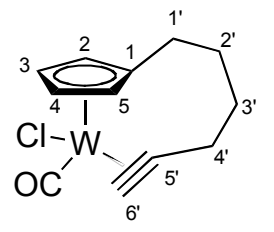

4 
by ${ }^{1} \mathrm{H}$ NMR spectroscopy and consumption of 2 was complete after $45 \mathrm{~min}$.

Yield of 4: $73 \%$ by ${ }^{1} \mathrm{H}$ NMR.

LR-MS (FAB): $m / z$ 355, 356, 357, 359 (matches M-Cl, $\mathrm{C}_{12} \mathrm{H}_{13} \mathrm{O}^{182,183,184,186} \mathrm{~W}$ ).

IR (THF): $v(\mathrm{CO}) 1924 \mathrm{~cm}^{-1}$.

${ }^{1} \mathrm{H}$ NMR (600.13 MHz, $\mathrm{C}_{6} \mathrm{D}_{6}$ ): $\delta 11.82$ (t (with ${ }^{183} \mathrm{~W}$ satellites), $\mathrm{J}_{6^{\prime}, 4^{\prime}}=1.0 \mathrm{~Hz}, \mathrm{~J}_{\mathrm{W}-\mathrm{H}}=5.2 \mathrm{~Hz}, 1 \mathrm{H}, \mathrm{H} 6^{\prime}$ ), 5.82 (dddt, $\mathrm{J}_{2,3}=3.2 \mathrm{~Hz}, \mathrm{~J}_{2,4}=1.9 \mathrm{~Hz}, \mathrm{~J}_{2,5}=1.9 \mathrm{~Hz}, \mathrm{~J}_{2,1}=0.6 \mathrm{~Hz}, 1 \mathrm{H}, \mathrm{H} 2$ ), 5.27 (dddd, $\mathrm{J}_{3,4}=3.3 \mathrm{~Hz}, \mathrm{~J}_{3,2}=3.2$ $\left.\mathrm{Hz}, \mathrm{J}_{3,5}=2.0 \mathrm{~Hz}, \mathrm{~J}_{3,1^{\prime} \mathrm{a}}=0.4 \mathrm{~Hz}, 1 \mathrm{H}, \mathrm{H} 3\right), 4.97\left(\mathrm{ddd}, \mathrm{J}_{4,3}=3.3 \mathrm{~Hz}, \mathrm{~J}_{4,5}=2.8 \mathrm{~Hz}, \mathrm{~J}_{4,2}=1.9 \mathrm{~Hz}, 1 \mathrm{H}, \mathrm{H} 4\right), 3.90$ (dddt, $\left.\mathrm{J}_{5,4}=2.8 \mathrm{~Hz}, \mathrm{~J}_{5,3}=2.0 \mathrm{~Hz}, \mathrm{~J}_{5,2}=1.9 \mathrm{~Hz}, \mathrm{~J}_{5,1}=0.5 \mathrm{~Hz}, 1 \mathrm{H}, \mathrm{H} 5\right), 3.45$ (dddd, $\mathrm{J}_{4^{\prime} \mathrm{a}, 4^{\prime} \mathrm{b}}=16.3 \mathrm{~Hz}, \mathrm{~J}_{4^{\prime} \mathrm{a}, 3^{\prime} \mathrm{a}}=8.3$ $\left.\mathrm{Hz}, \mathrm{J}_{4^{\prime} \mathrm{a}, 3^{\prime} \mathrm{b}}=2.7 \mathrm{~Hz}, \mathrm{~J}_{4^{\prime} \mathrm{a}, 6^{\prime}}=1.0 \mathrm{~Hz}, 1 \mathrm{H}, \mathrm{H} 4^{\prime} \mathrm{a}\right), 3.05\left(\mathrm{dddd}, \mathrm{J}_{4^{\prime} \mathrm{b}, 4^{\prime} \mathrm{a}}=16.3 \mathrm{~Hz}, \mathrm{~J}_{4^{\prime} \mathrm{b}, 3^{\prime} \mathrm{b}}=10.0 \mathrm{~Hz}, \mathrm{~J}_{4^{\prime} \mathrm{b}, 3^{\prime} \mathrm{a}}=2.4 \mathrm{~Hz}\right.$, $\left.\mathrm{J}_{4^{\prime} \mathrm{b}, 6^{\prime}}=1.0 \mathrm{~Hz}, 1 \mathrm{H}, \mathrm{H} 4^{\prime} \mathrm{b}\right), 2.39\left(\mathrm{dddt}, \mathrm{J}_{1^{\prime} \mathrm{b}, 1^{\prime} \mathrm{a}}=16.4 \mathrm{~Hz}, \mathrm{~J}_{1^{\prime} \mathrm{b}, 2^{\prime} \mathrm{b}}=7.4 \mathrm{~Hz}, \mathrm{~J}_{1^{\prime} \mathrm{b}, 2^{\prime} \mathrm{a}}=3.0 \mathrm{~Hz}, \mathrm{~J}_{1^{\prime} \mathrm{b}, 2 / 5}=0.6 \mathrm{~Hz}, 1 \mathrm{H}\right.$, H1'b), 1.81 (ddddd, $J_{3^{\prime} b, 3^{\prime} \mathrm{a}}=14.4 \mathrm{~Hz}, \mathrm{~J}_{3^{\prime} \mathrm{b}, 4^{\prime} \mathrm{b}}=10.0 \mathrm{~Hz}, \mathrm{~J}_{3^{\prime} \mathrm{b}, 2^{\prime} \mathrm{b}}=9.7 \mathrm{~Hz}, \mathrm{~J}_{3^{\prime} \mathrm{b}, 2^{\prime} \mathrm{a}}=3.6 \mathrm{~Hz}, \mathrm{~J}_{3^{\prime} \mathrm{b}, 4^{\prime} \mathrm{a}}=2.7 \mathrm{~Hz}, 1 \mathrm{H}$, H3'b), 1.61 (ddddd, $\mathrm{J}_{2^{\prime} \mathrm{a}, 2^{\prime} \mathrm{b}}=14.8 \mathrm{~Hz}, \mathrm{~J}_{2^{\prime} \mathrm{a}, 1^{\prime} \mathrm{a}}=10.9 \mathrm{~Hz}, \mathrm{~J}_{2^{\prime} \mathrm{a}, 3^{\prime} \mathrm{a}}=7.1 \mathrm{~Hz}, \mathrm{~J}_{2^{\prime} \mathrm{a}, 3^{\prime} \mathrm{b}}=3.6 \mathrm{~Hz}, \mathrm{~J}_{2^{\prime} \mathrm{a}, 1^{\prime} \mathrm{b}}=3.0 \mathrm{~Hz}, 1 \mathrm{H}$, H2'a), 1.30 (dddddd, $\mathrm{J}_{1^{\prime} \mathrm{a}, 1^{\prime} \mathrm{b}}=16.4 \mathrm{~Hz}, \mathrm{~J}_{1^{\prime} \mathrm{a}, 2^{\prime} \mathrm{a}}=10.9 \mathrm{~Hz}, \mathrm{~J}_{1^{\prime} \mathrm{a}, 2^{\prime} \mathrm{b}}=2.9 \mathrm{~Hz}, \mathrm{~J}_{1^{\prime} \mathrm{a}, 2}=0.6 \mathrm{~Hz}, \mathrm{~J}_{1^{\prime} \mathrm{a}, 5}=0.5 \mathrm{~Hz}, \mathrm{~J}_{1^{\prime} \mathrm{a}, 3}=0.4$ $\mathrm{Hz}, 1 \mathrm{H}, \mathrm{H} 1^{\prime} \mathrm{a}$ ), 1.27 (ddddd, $\mathrm{J}_{3^{\prime} \mathrm{a}, 3^{\prime} \mathrm{b}}=14.4 \mathrm{~Hz}, \mathrm{~J}_{3^{\prime} \mathrm{a}, 4^{\prime} \mathrm{a}}=8.3 \mathrm{~Hz}, \mathrm{~J}_{3^{\prime} \mathrm{a}, 2^{\prime} \mathrm{a}}=7.1 \mathrm{~Hz}, \mathrm{~J}_{3^{\prime} \mathrm{a}, 2^{\prime} \mathrm{b}}=3.9 \mathrm{~Hz}, \mathrm{~J}_{3^{\prime} \mathrm{a}, 4^{\prime} \mathrm{b}}=2.4 \mathrm{~Hz}$, $1 \mathrm{H}, \mathrm{H} 3^{\prime} \mathrm{a}$ ), 1.15 (ddddd, $\mathrm{J}_{2^{\prime} \mathrm{b}, 2^{\prime} \mathrm{a}}=14.8 \mathrm{~Hz}, \mathrm{~J}_{2^{\prime} \mathrm{b}, 3^{\prime} \mathrm{b}}=9.7 \mathrm{~Hz}, \mathrm{~J}_{2^{\prime} \mathrm{b}, 1^{\prime} \mathrm{b}}=7.4 \mathrm{~Hz}, \mathrm{~J}_{2^{\prime} \mathrm{b}, 3^{\prime} \mathrm{a}}=3.9 \mathrm{~Hz}, \mathrm{~J}_{2^{\prime} \mathrm{b}, 1^{\prime} \mathrm{a}}=2.9 \mathrm{~Hz}, 1 \mathrm{H}$, $\mathrm{H} 2 \mathrm{\prime} b)$.

${ }^{13} \mathrm{C}$ NMR (150.9 MHz, $\left.\mathrm{C}_{6} \mathrm{D}_{6}\right): \quad \delta 228.22$ (CO), 202.49 (C5'), 179.55 (C6'), 113.48 (C1), 109.13 (C3), $102.36(\mathrm{C} 2), 84.98(\mathrm{C} 4), 82.17(\mathrm{C} 5), 35.97\left(\mathrm{C} 4{ }^{\prime}\right), 27.28\left(\mathrm{C}^{\prime}\right), 26.52\left(\mathrm{C} 3^{\prime}\right), 25.60\left(\mathrm{C} 2{ }^{\prime}\right)$.

\section{References}

(1) Eglinton, G.; Whiting, M. C. J. Chem. Soc. 1950, 3650.

(2) Newman, M. S.; Wotiz, J. H. J. Am. Chem. Soc. 1949, 71, 1292-1297.

(3) Alder, K.; Ache, H.-J. Chem. Ber. 1962, 95, 503-510. 